\title{
A new MV bus transfer scheme for nuclear power plants
}

\author{
Choong-Koo Chang* \\ Department of NPP Engineering, KEPCO International Nuclear Graduate School (KINGS), Ulsan, Korea
}

Received: 29 April 2015 / Received in final form: 2 September 2015 / Accepted: 12 October 2015

Published online: 09 December 2015

\begin{abstract}
Fast bus transfer method is the most popular and residual voltage transfer method that is used as a backup in medium voltage buses in general. The use of the advanced technology like open circuit voltage prediction and digital signal processing algorithms can improve the reliability of fast transfer scheme. However, according to the survey results of the recent operation records in nuclear power plants, there were many instances where the fast transfer scheme has failed. To assure bus transfer in any conditions and circumstances, uninterruptible bus transfer scheme utilizing the state of the art medium voltage UPS is discussed and elaborated.
\end{abstract}

\section{Introduction}

The auxiliary power system of many generating stations consists of offsite power supply system and onsite power supply system, including emergency diesel generators (EDG) to provide secure power to auxiliary loads. If a normal power supply fails to supply power, then the power source is transferred to a standby power supply. In the case of nuclear power plants (NPP), the unit auxiliary transformer (UAT) and standby auxiliary transformer (SAT) - or station service transformer - are installed and powered from two offsite power circuits to meet regulatory requirements (10 CFR 50, App. A). Figure 1 is a simplified single line diagram of the auxiliary power system for APR 1400 type nuclear power plant. The transfer methods of a motor bus from a normal source to a standby source used in power generating stations are fast bus transfer, in-phase transfer, or residual transfer. Three important parameters, which are crucial from a bus transfer point of view, are the magnitude of the residual voltage, decay time, and the associated phase angle of the residual voltage [1]. The problem is, if the parameters do not meet the transfer conditions, the bus transfer will fail. Therefore, a new MV (medium voltage) bus transfer scheme is presented in this paper. It guarantees a successful bus transfer regardless of the bus condition.

\section{$2 \mathrm{MV}$ bus transfer requirements in NPP}

\subsection{Reactor coolant pump motor buses}

Both of the reactor coolant loops should be available and two (2) reactor coolant pumps of each loop should be in

\footnotetext{
*e-mail: ckchang@kings.ac.kr
}

operation for the normal operation of the reactor in pressurized water reactors. If any one (1) of four (4) pumps is inoperable, then the operation mode should be changed to the hot standby mode from normal operation mode. It means that the fast transfer is essential to the RCP motor buses (Divisions I and II) to maintain reactor operation. Typical transfer time of the fast transfer is 4 to 9 cycles. If both of the RCP motor buses fail to transfer, the reactor must go into the hot shutdown and cold shutdown mode successively. If a reactor is shut down, it takes a long time to restart generation. The time required to reach full power from the hot-tripped condition is around 4 to 6 hours. And, the minimum time required for starting up large LWRs (light water reactors) from the cold tripped condition may be around 20 hours [2].

\subsection{Medium voltage safety (class $1 E)$ bus}

The preferred power supply (PPS) to the safety power systems is from the grid. During power operation, the power supply is normally from the main generator connected to the grid. The offsite power should be supplied by two or more physically independent offsite supplies designed and located in order to minimize the likelihood of their simultaneous failure. A minimum of one offsite circuit should be designed to be automatically available to provide power to its associated safety divisions within a few seconds, following a design basis accident to meet the accident analysis requirements. A second offsite circuit should be designed to be available within a short time period [3].

The load capacity of the safety bus varies with the operation mode of the plant. Therefore, the most practical bus transfer method of MV class $1 \mathrm{E}$ buses is residual voltage 


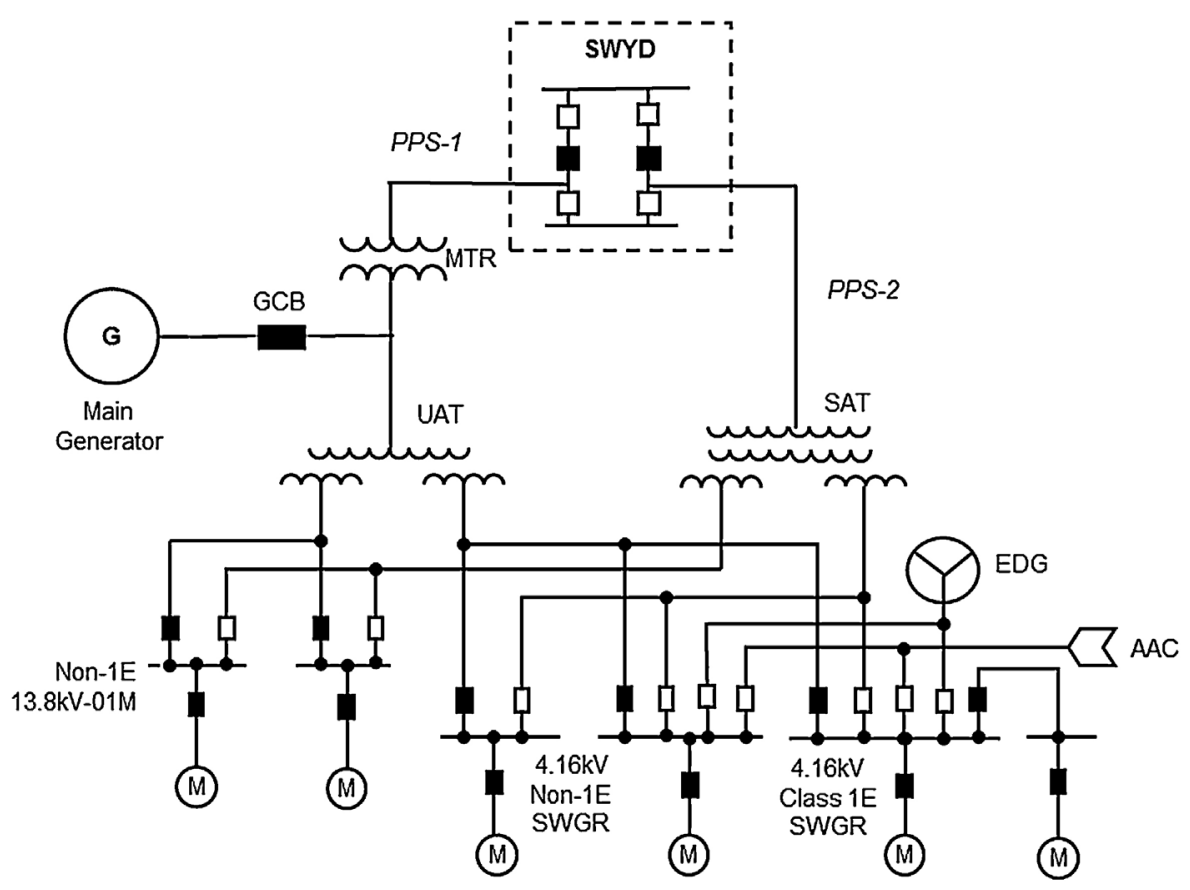

Fig. 1. Simplified MV bus single line diagram (Division I).

transfer. Furthermore, to avoid the alternative source overloading in the case of motors' low voltage restarting, it is required to implement low voltage load-shedding function before the residual voltage transfer. Typical transfer time of residual transfer is 0.5 to 3 seconds.

Each division of the MV class $1 \mathrm{E}$ power system is supplied with emergency standby power from an independent EDG. In the case of APR 1400, if the loss of voltage is detected by four time delay type undervoltage relays, the EDG is started to attain rated voltage and frequency within 20 seconds after receipt of a start signal, then supply power to $4.16 \mathrm{kV}$ AC class $1 \mathrm{E}$ bus within 22 seconds.

\section{Bus transfer methods in general}

There are several bus transfer methods used to transfer a MV motor bus from a normal source to a standby source. The detail features and functions of a bus transfer device are dependent on manufacturers. However, basic concept is the same and a typical MV bus transfer scheme is as follows [4,5].

\subsection{Fast transfer}

In the auxiliary power system of power stations and industrial plants, lots of asynchronous motors are connected. In the case of the main source interruption, the residual voltage will be induced on the busbar by connected asynchronous motors. Studies to determine the magnitude of the transient current and torque are recognized to be complex and require detailed knowledge of the motor, the driven equipment, and the power supply.

\subsubsection{Sequential transfer}

Under the sequential transfer, a bus transfer device will firstly issue an open command to the running source CB (circuit breaker) after the device gets the starting request command. Sequential transfer can only issue close command after the running source CB is opened. The switching sequence of the sequential transfer scheme is illustrated in Figure 2 [5]. This approach provides increased security because the bus has been disconnected from the main source prior to the standby source breaker closing. A bus dead time of $5 \sim 7$ cycles is normally encountered with this type of transfer $[6,7]$.

\subsubsection{Simultaneous transfer}

If two sources are not allowed to work on a busbar in parallel, the simultaneous sequence can be used for the power supply transfer [7].

Under the simultaneous sequence, a bus transfer device will firstly issue an open command to the running source CB after the device gets the starting command. Meanwhile, the device will issue a close command to the standby source CB if criteria are met [5]. This type of transfer has the shortest dead time of $1 \sim 2$ cycles. This type of transfer may not be possible when the main source is lost due to a close-in electrical fault or abnormal condition that causes the phase angle to move instantaneously. Also, a breaker failure scheme is required for the simultaneous transfer so that if the main source breaker fails to open during the transfer, then the standby source breaker is tripped to avoid paralleling of two sources [6]. 


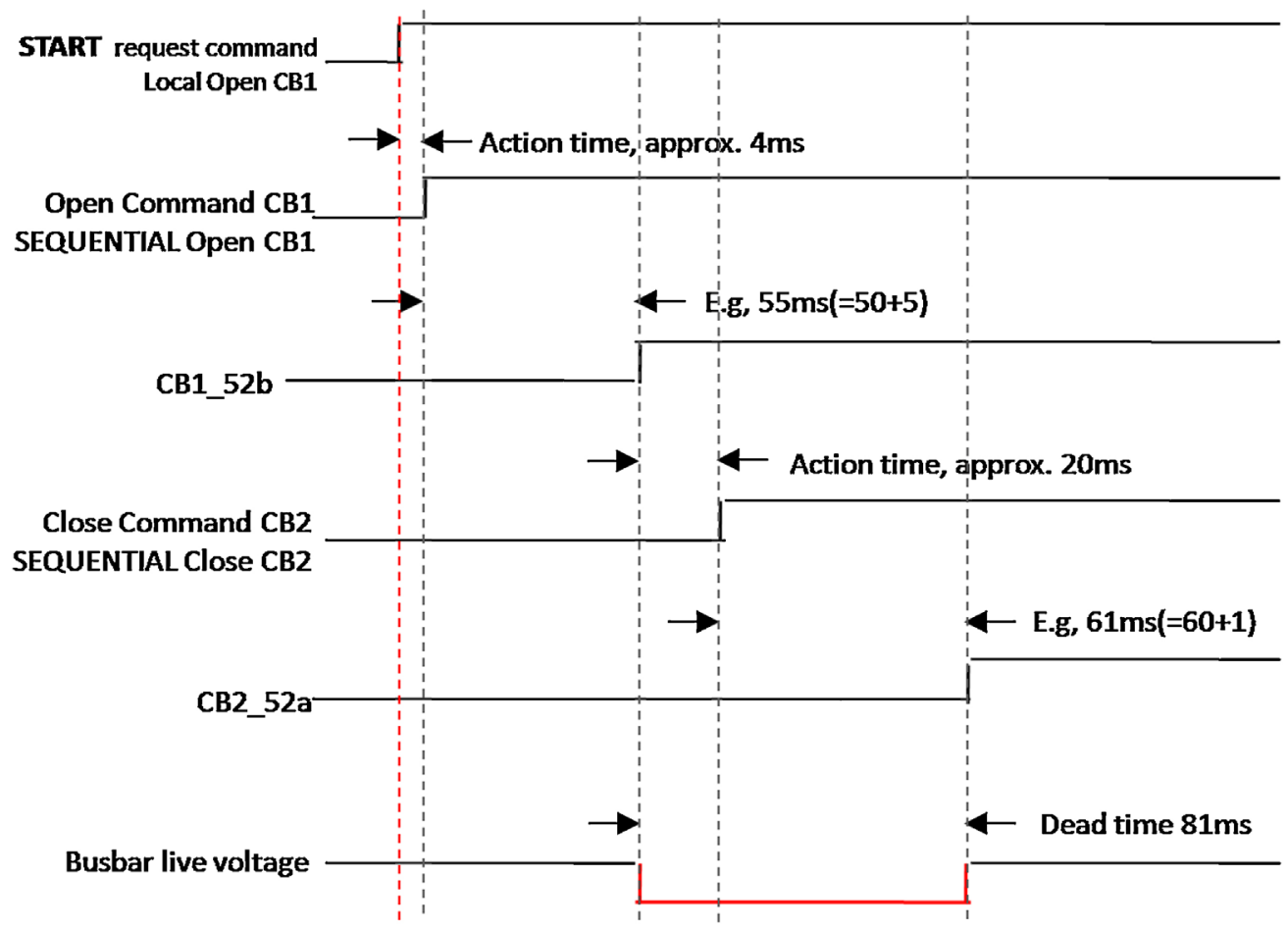

Fig. 2. Switching sequence illustration of sequential transfer.

\subsection{In-phase transfer}

In the in-phase transfer scheme, the standby source breaker is closed when the phase angle of the bus voltage is in-phase with the phase angle of the standby source voltage. The bus transfer device estimates the phase angle difference $d \varphi$ at the instance of CB closing based on realtime slipping rate and the settable "CB Closing Time". If all the quantity of predicted $d \varphi$, the real-time $d f$, and residual voltage $U_{\text {res }}$ meet the defined criteria, the device will immediately issue the close command to the alternative source CB $[5,7]$.

\subsection{Residual voltage transfer}

If the above-mentioned transfer modes fail, the transfer can still go on with residual voltage transfer mode. When the residual voltage $U_{\text {res }}$ under-shots the settable parameter " $U_{\text {res }}$ Threshold", the residual voltage transfer mode will perform and the device will immediately issue the close command to the standby source CB. The typical setting could be $30 \%$ of rated bus voltage $U_{n}[5]$. Typically, residual voltage transfers are done at $25 \% \sim 30 \%$ of the rated voltage, irrespective of the phase angle of the motor bus. As the residual transfer is slow, process interruption is likely to take place. Also, in the majority of cases, motors cannot be reaccelerated simultaneously following such a transfer as their speeds have fallen so low that inrush currents approach motor locked-rotor values, and stalling would occur due to depressed voltage [8].

\section{Challenges in MV bus transfer system}

Research and development on the MV bus transfer system has been continuing since 1950s to meet the necessity of highly reliable power system in the industry and power plants [8]. Especially, in a nuclear power plant, reliable power supply is essential for the safe operation of the power plant and the prevention of the release of radioactive fission products. However, successful bus transfer was not assured until now; although, there were remarkable achievements in the development of improved fast bus transfer system.

\subsection{Typical recent research results and achievements}

To ensure the success of fast bus transfer, many research activities had been conducted. Typical recent research results and achievements are as follows.

\subsubsection{Prediction of open circuit voltage}

The open circuit characteristic of a motor bus auxiliary is influenced by the type of motors selected, breakdown torque of induction motors, load characteristics, and motor inertia. The type of bus transfer - such as sequential fast transfer or in-phase transfer of the motor bus - is also influenced by the above factors. The estimation of the open circuit time constant of the motor bus is therefore critical to 
accurately predict how the voltage and frequency of the motor bus is going to decay. A successful bus transfer depends on the thorough understanding of the process and a proper analysis of the auxiliary system using proper simulation tools. A robust bus transfer technique is presented in the Balamourougan's paper which determines, in approximately one cycle after the motor bus has been interrupted, whether a sequential fast bus transfer is possible or not by estimating the rate of decay of the motor bus residual voltage, magnitude of residual voltage, and the phase angle [1].

\subsubsection{Digital signal processing algorithms}

The design of a digital high-speed bus transfer system has been presented in the Yalla's and Sidhu's paper [6]. Digital signal processing algorithms that calculate the magnitude and phase angle of a voltage signal over a wide frequency range have been presented. Also presented is an algorithm to predict the phase coincidence between the motor bus voltage and the standby source voltage using delta frequency, the rate of change of delta frequency, and breaker closing time, which results in a very accurate prediction of phase coincidence. The algorithm has also calculated the bus voltage magnitude very accurately down to $4 \mathrm{~Hz}$, which is important for the residual transfer method.

\subsubsection{Advanced modeling and digital simulation}

Presently, advanced modeling and digital simulation tools help in modeling and simulation of complex fast transfer scheme. They are used to analyze whether the conditions are met to adopt fast transfer with the realistic loads. The preliminary assessment assures successful fast transfer for the estimated transfer time and the inertia constants based on the preliminary data sheets from the vendors and the generic modeling of turbine generator excitation and governing system [8].

\subsubsection{Implementation of IEC 61850 standard}

The new communications technology and the newly developed International Electrotechnical Commission (IEC) standard IEC 61850, for generic object-oriented substation events (GOOSE), bring many advantages to industrial protection and control applications. Some of the applications benefiting the most are those associated with the bus-transfer and load-shedding schemes, together with more beneficial communication-assisted schemes, like zone interlocking, fast bus trip, and arc-flash reduction. A fast bus trip scheme using GOOSE messaging is performed by the relays from the main breakers without the need of adding a bus differential relay. In such cases, relays from the main breakers are connected via fiber optic or copper twisted pair Ethernet cables to the entire feeder relays to exchange GOOSE data [10].

\subsection{Causes of the failure of bus transfer schemes in nuclear power plants}

Fast bus transfer method is the most popular and residual voltage transfer method that is used as a backup in nuclear power plants of Korea. During the last 10 years (2005 2014), 30 cases of reactor shutdown events caused by electrical faults had been reported by Korea Institute of Nuclear Safety [11]. Among them, 20 cases are the events for which fast bus transfer (or residual voltage transfer) is required. However, there was only one successful bus transfer. In the other 12 cases, the bus transfer had failed. No information is available for the remaining seven cases. Major reasons of bus transfer failure are as follows and summarized in Table 1.

\subsubsection{Malfunction of circuit breaker}

The malfunction of circuit breaker closing device caused the failure of the bus transfer. Accordingly, RCPs were stopped and followed by reactor shutdown.

\subsubsection{Ground fault on the normal source}

The ground fault occurring on the low voltage side of the Main Transformer made low bus voltage. Accordingly, fast bus transfer to alternative source was unsuccessful. In the other case, the ground fault occurred on the secondary side of the Startup Transformer which caused loss of class 1E bus voltage. Then EDG was started automatically.

\subsubsection{Perturbation of grid voltage}

Bus transfer was initiated when a grid lost load-following capability. It caused voltage dip and phase angle change. Accordingly, bus transfer was unsuccessful.

\subsubsection{Other unidentified failures}

Causes of some bus transfer failures were not verified clearly. But it is assumed that fast bus transfer or residual bus transfer requirements were not satisfied by any reason.

Table 1. Types of bus transfer failure.

\begin{tabular}{lcl}
\hline Cause & Number & Remark \\
\hline CB malfunction & 4 & Failure of closing \\
$\begin{array}{l}\text { Ground fault } \\
\text { Perturbation }\end{array}$ & 2 & Low bus voltage \\
of grid voltage & 1 & Low bus voltage \\
Others & 5 & $\begin{array}{l}\text { Dissatisfaction of } \\
\text { bus transfer conditions }\end{array}$ \\
Total & 12 & \\
\hline
\end{tabular}




\section{Uninterruptible MV bus transfer scheme}

To guarantee the safety of nuclear power plants, interruption of power supply shall be prevented. For the purpose of satisfying such requirements, emergency power systems are provided including EDGs, DC batteries, and vital AC power supplies. Nowadays, it is possible to reach almost $100 \%$ availability of power supply for low voltage systems. However, there is still a gap when facing applying these emergency power systems in medium voltage systems, for several reasons: investment, space, long-term energy losses cost, high temperatures or dirtiness, and regenerative loads. To resolve this problem, uninterruptible MV bus transfer scheme is proposed by combination of existing bus transfer system and industrial UPS (uninterruptible power supply). In general, the fast transfer of $13.8 \mathrm{kV}$ bus is more successful than $4.16 \mathrm{kV}$ bus because the RCP motors fed from $13.8 \mathrm{kV}$ bus have high inertia and are large size. Furthermore, $4.16 \mathrm{kV}$ class $1 \mathrm{E}$ loads are more critical than $13.8 \mathrm{kV}$ loads in terms of safety. All reactor coolant pumps may be de-energized for up to $\leq 1$ hour per 8-hour period, provided: (a) no operations are permitted that would cause reduction of the RCS boron concentration; and (b) core outlet temperature is maintained at least $5.6{ }^{\circ} \mathrm{C}$ $\left(10^{\circ} \mathrm{F}\right)$ below saturation temperature [12]. Therefore, uninterruptible MV transfer system is proposed for $4.16 \mathrm{kV}$ class $1 \mathrm{E}$ bus. And, the available capacity of energy storage device also has been considered.

\subsection{Existing class $1 \mathrm{E} 4.16 \mathrm{kV}$ buses operation scheme}

Each division of the $4.16 \mathrm{kV}$ AC class $1 \mathrm{E}$ power system is supplied with emergency standby power from an independent EDG. The EDG is designed and sized with sufficient capacity to operate all the needed emergency shutdown loads powered from its respective class $1 \mathrm{E}$ buses. Each EDG is designed to attain rated voltage and frequency within
20 seconds after receipt of a start signal, supply power to $4.16 \mathrm{kV}$ AC class $1 \mathrm{E}$ bus within 22 seconds [12].

Degraded voltage is detected by the time delay type relay of which the setting is higher than the setting value of the undervoltage relay for loss of voltage and lower than the required minimum operating voltage. A detection signal is provided to ESF-CCS (Engineered Safety Feature-Component Control System). The EDG is started on ESF actuation signal, and ready for operation. However, the EDG is not connected to $4.16 \mathrm{kV}$ class $1 \mathrm{E}$ bus when normal or standby power is available but remains in standby. The class $1 \mathrm{E}$ loads are powered from the normal or standby power source.

When the loss of voltage is detected, if the standby power is available, the $4.16 \mathrm{kV}$ class $1 \mathrm{E}$ buses are transferred to standby power source by using residual voltage transfer scheme with the shedding of non-class $1 \mathrm{E}$ loads. In case of bus transfer failure, the EDG is started by undervoltage on the bus, all breakers for $4.16 \mathrm{kV}$ motor feeders are tripped and the load sequencer is reset. Upon detection of the EDG rated speed and rated voltage, the EDG circuit breakers on class $1 \mathrm{E}$ $4.6 \mathrm{kV}$ bus can be closed and the load sequence logic starts automatically, sequencing the safety related loads on the EDG. The required safety related loads are connected to the bus in the preselected interval time. Thus, the EDG can be operated stable and minimize motor acceleration time.

Each EDG and the automatic sequencers necessary for generator loading are designed such that flow is delivered to the reactor vessel within a maximum of 40 seconds after an SIAS set point is reached [12].

\subsection{Implementation of MV UPS on the class 1E $4.16 \mathrm{kV}$ buses in a NPP}

By applying a MV UPS on the class 1E switchgear incoming feeder as shown in Figure 3, seamless transfer can be achieved without shedding of loads. When loss of voltage is

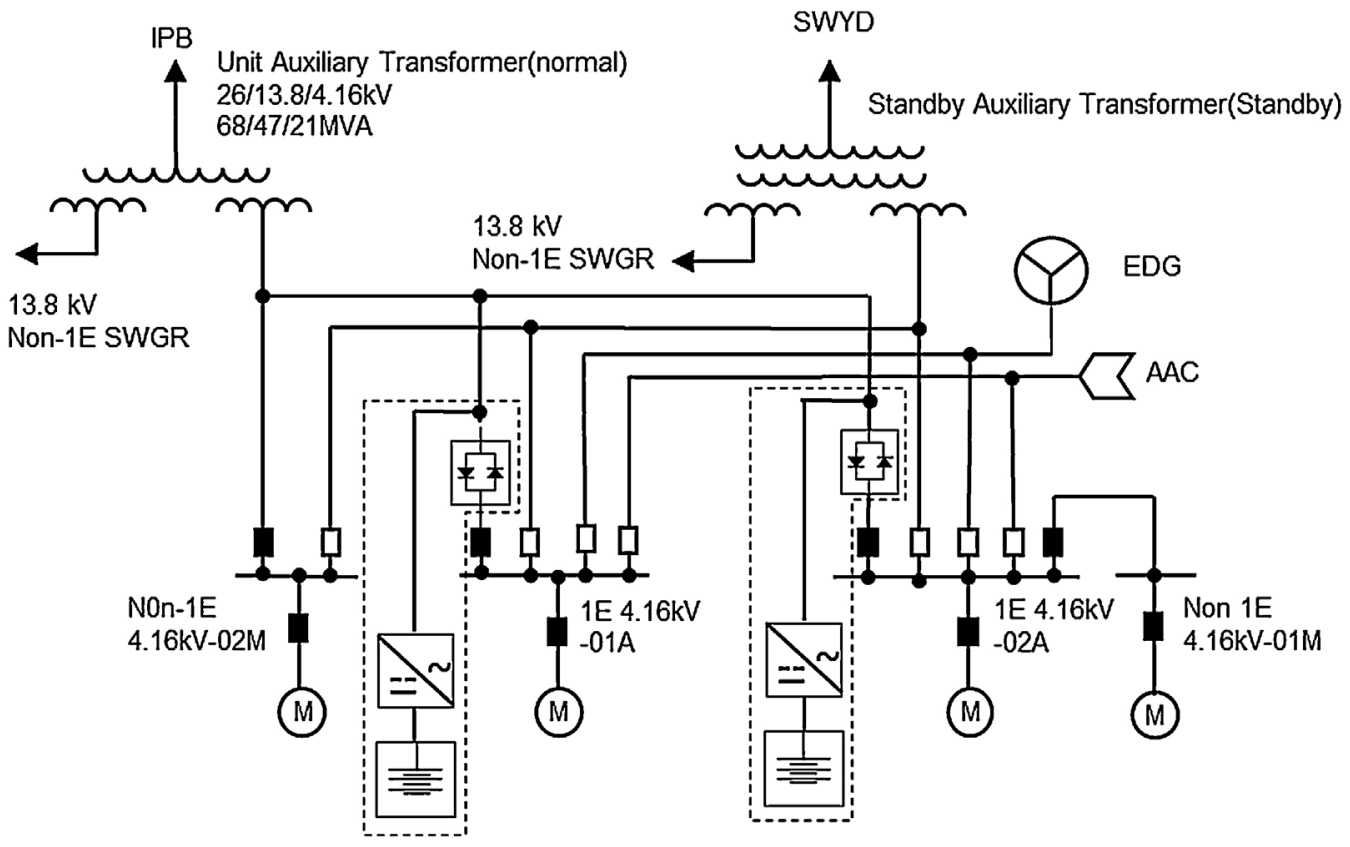

Fig. 3. $4.16 \mathrm{kV}$ buses of a nuclear power plant (Division I only). 


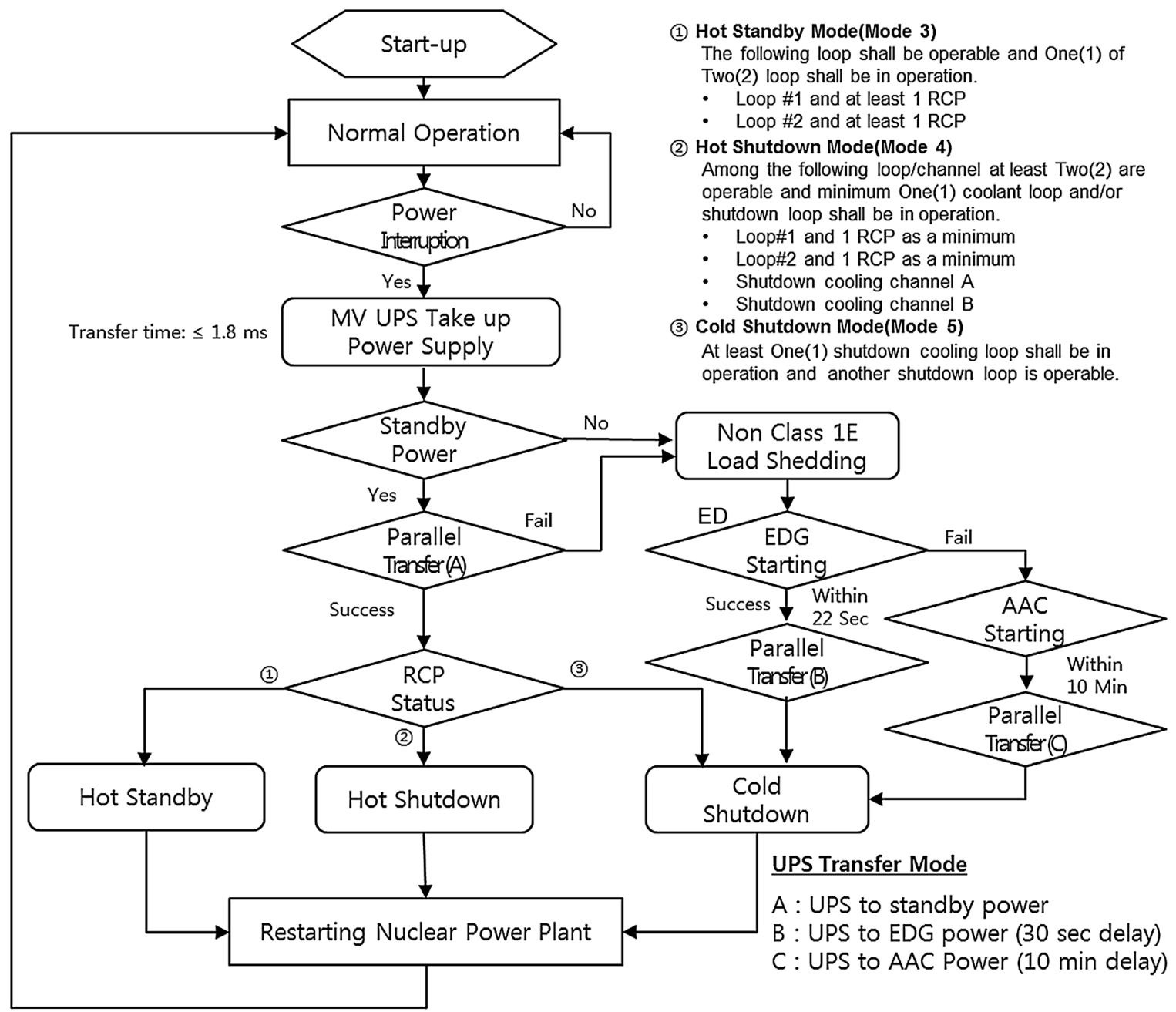

Fig. 4. Uninterruptible MV bus transfer system functional flow diagram.

occurred in the normal source, if the standby power source is available, the class $1 \mathrm{E}$ bus is transferred to the standby power source with the momentary backup of the UPS without interruption of power supply to the bus.

The flow of uninterruptible bus transfer scheme is shown in Figure 4.

When the loss of both normal and standby source is detected, the UPS supply power to the class $1 \mathrm{E}$ bus until EDG is initiated and supplies power to the class $1 \mathrm{E}$ bus for about 30 seconds. Therefore, the power of class $1 \mathrm{E} 4.16 \mathrm{kV}$ bus is not interrupted.

If the EDG also fails to start, then the Alternative AC (AAC) generator is started manually within 10 minutes. Until the AAC generator starts, the UPS supplies power to the $4.16 \mathrm{kV}$ class $1 \mathrm{E}$ buses. As a result, power supply to nuclear safety systems is not interrupted all the time. It means that motors are not needed to stop and restart during the bus transfer.

\subsection{Medium voltage UPS}

In case of power supply loss, the MV UPS operates disconnected from the normal power but continues to supply loads (Fig. 5). Then, the MV UPS synchronizes the islanded bus to the standby source. Estimated capacity of the MV UPS for the Division I, class $1 \mathrm{E}$ buses is approximately 10 MVA each. The MV UPS supports the load when the voltage is outside a user set window. Targeted protections are voltage sags, voltage swells, and short and long outages $[9,13]$.

\subsubsection{Converter module}

MV UPS uses the LV power modules which employ IGBT's and integrated sinusoidal filters. Multiple modules are connected in parallel to provide higher power. The modules are current rated and the available power output depends on the $\mathrm{AC}$ coupling voltage. The AC coupling voltage is further defined by the lowest possible DC link voltage. This is the minimum operational or discharging voltage of the storage.

\subsubsection{Energy storage system}

The MV UPS is designed by using energy storage device. The most common are super capacitors, lithium-ion 


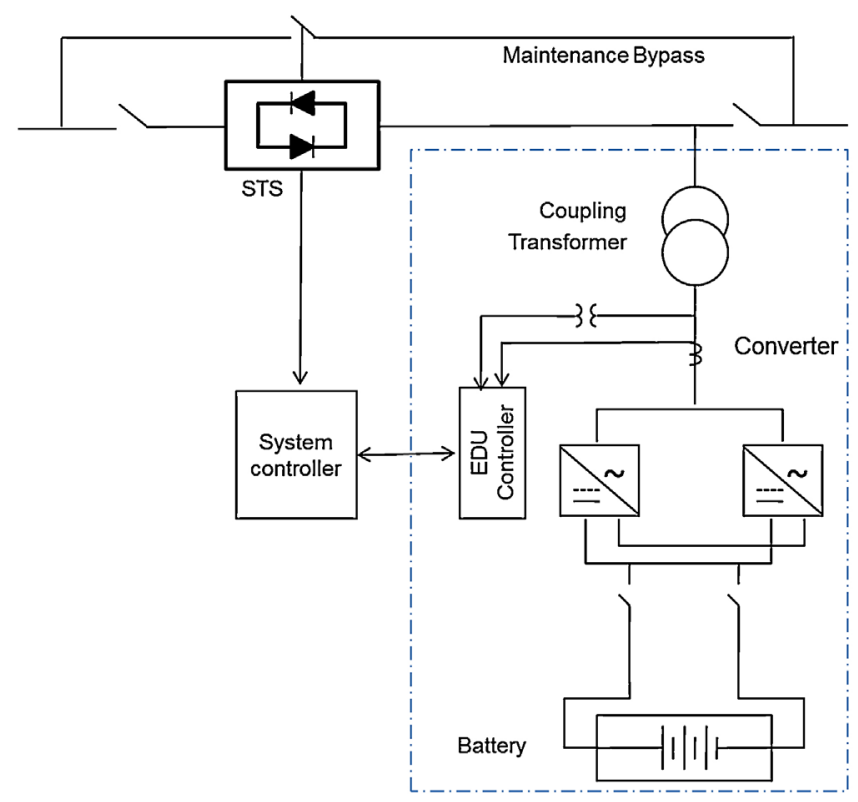

Fig. 5. Schematic diagram of MV UPS.

batteries, and high-discharge sealed lead-acid batteries. It is expected that super capacitors will be widely used in industrial applications due to their long life and compact size. For longer-autonomy applications, lithium-ion batteries similar to those used in electric cars offer reduced footprint and increased life when compared with the lowestcost, lead-acid solutions. Lithium-ion batteries have excellent cycle life characteristics.

\subsubsection{Coupling transformer}

The AC connection voltage of the MV UPS depends on the batteries used. Therefore, usually a coupling transformer is needed to obtain required bus voltage.

\subsubsection{Control}

The MV UPS controls its own voltage and frequency, enabling it to create a micro or islanded grid. When the motor bus is disconnected from the utility, the MV UPS will support the MV bus loads with minimal disturbance. The monitoring and indicating of a normal source failure can be done externally or by internal supervision based on frequency/voltage monitoring. After confirming the standby source, the MV UPS can synchronize the MV bus to the standby source.

\subsubsection{Technical specifications and rating of MV UPS}

Table 2 shows the typical technical specifications of the $4.16 \mathrm{kV}$ UPS for the Division I.

The power converter and energy storage remain at low voltage, with a transformer coupling these to medium voltage. Also at the medium voltage level is a thyristor-based disconnection switch which prevents backfeed into the grid in the event of power loss or voltage sag. The MV UPS is compatible with a wide range of energy storage depending on the duration of protection required. Super capacitors and flywheels provide high-density coverage for seconds while batteries can be used for longer backup times for up to 15 minutes. The 10 MVA MV UPS is recommended for the $4.16 \mathrm{kV}$ class $1 \mathrm{E}$ buses considering the maximum loads safety margin. As shown in Figure 3, class 1E bus 02A and Non-1E bus $01 \mathrm{M}$ are interconnected. Therefore, the MV UPS shall be sized to include bus 01M load.

\subsubsection{Selection of battery type and sizing capacity}

Energy storage devices applicable to MV UPS are super capacitors, lead-acid batteries, lithium-ion batteries, and flywheels. Characteristics of each solution are as specified in Table 3. Among them, lithium-ion battery is preferred to be used for the MV UPS. The energy density of lithium-ion battery is three to five times that of the lead-acid stationary

Table 2. Technical specifications of MV UPS.

\begin{tabular}{|c|c|c|c|c|}
\hline \multicolumn{2}{|l|}{ Item } & \multicolumn{3}{|c|}{ Specification } \\
\hline \multicolumn{2}{|l|}{ Nominal voltage } & \multicolumn{3}{|c|}{$4.16 \mathrm{kV} \pm 10 \%$} \\
\hline \multicolumn{2}{|l|}{ Rating } & \multicolumn{3}{|c|}{$10 \mathrm{MW}$} \\
\hline \multicolumn{2}{|l|}{ Efficiency (full load) } & \multicolumn{3}{|c|}{$99.5 \%$} \\
\hline \multicolumn{2}{|l|}{ Autonomy } & \multicolumn{3}{|c|}{ Up to $15 \mathrm{~min}$} \\
\hline \multicolumn{2}{|l|}{ Displacement power FACTOR } & \multicolumn{3}{|c|}{0.7 lagging to 0.9 leading } \\
\hline \multicolumn{2}{|l|}{ Typical transfer time } & \multicolumn{3}{|c|}{$\leq 1.8 \mathrm{~ms}$ (typical) } \\
\hline \multicolumn{2}{|l|}{ UPS footprint } & \multicolumn{3}{|c|}{$54 \mathrm{~m}^{2}$ (without storage) } \\
\hline \multicolumn{2}{|l|}{ System DC nominal voltage } & \multicolumn{3}{|c|}{$750 \mathrm{~V} \mathrm{DC}(812 \sim 554 \mathrm{~V}$ DC $)$} \\
\hline \multicolumn{5}{|c|}{ The MV UPS may be equipped with one of three storage devices } \\
\hline Energy storage & Volt/Cell & $\mathrm{AH}$ & Autonomy & Footprint \\
\hline Super capacitor & - & - & $1 \mathrm{~s}$ & $10 \mathrm{~m}^{2}$ \\
\hline Lead-acid & $12 \mathrm{~V}$ & 50 & $30 \mathrm{~s}$ & $25.6 \mathrm{~m}^{2}$ \\
\hline Lithium-ion & $3.7 \mathrm{~V}$ & 60 & $15 \min$ & $42 \mathrm{~m}^{2}$ \\
\hline
\end{tabular}


Table 3. Comparisons of energy storage devices.

\begin{tabular}{lllll}
\hline Item & Super capacitor & Lead-acid battery & Lithium-ion battery & Flywheel \\
\hline Energy density & High & Standard & High & - \\
Design life & 15 years & 10 years & 20 years & Long with maintenance \\
Autonomy & $1 \mathrm{~s}$ & $30 \mathrm{~s} \sim 15 \mathrm{~min}$ & $30 \mathrm{~s} \sim 15 \mathrm{~min}$ & $10 \sim 15 \mathrm{~s}$ \\
Ambient temperature & $25^{\circ} \mathrm{C}$ & $25{ }^{\circ} \mathrm{C}$ & $40^{\circ} \mathrm{C}$ & - \\
Etc. & High discharge & - & - & Very high cycle life \\
\hline
\end{tabular}

Table 4. Load capacities at normal operation.

\begin{tabular}{llllll}
\hline Description & Non-1E & Non-1E & Class 1E & Class 1E & $02 \mathrm{~A}+01 \mathrm{M}$ \\
& $4.16 \mathrm{kV}-01 \mathrm{M}$ & $4.16 \mathrm{kV}-02 \mathrm{M}$ & $4.16 \mathrm{kV}-01 \mathrm{~A}$ & $4.16 \mathrm{kV}-02 \mathrm{~A}$ & \\
\hline Apparent (MVA) & 1.97 & 5.85 & 5.17 & 1.38 & 3.35 \\
Active (MW) & 1.74 & 5.04 & 4.62 & 1.19 & 2.93 \\
Reactive (Mvar) & 0.92 & 2.96 & 2.32 & 0.70 & 1.62 \\
\hline
\end{tabular}

Table 5. Load capacities at LOCA mode.

\begin{tabular}{llllll}
\hline Description & Non-1E & Non-1E & Class 1E & Class 1E & $02 \mathrm{~A}+01 \mathrm{M}$ \\
& $4.16 \mathrm{kV}-01 \mathrm{M}$ & $4.16 \mathrm{kV}-02 \mathrm{M}$ & $4.16 \mathrm{kV}-01 \mathrm{~A}$ & $4.16 \mathrm{kV}-02 \mathrm{~A}$ & \\
\hline Apparent (MVA) & 0.54 & 5.85 & 6.79 & 2.82 & 3.36 \\
Active (MW) & 0.46 & 5.04 & 6.08 & 2.49 & 2.95 \\
Reactive (Mvar) & 0.28 & 2.96 & 3.03 & 1.33 & 1.41 \\
\hline
\end{tabular}

battery. Furthermore, lithium-ion battery is a low maintenance battery, an advantage that most other chemistries cannot claim. There is no memory and no scheduled cycling is required to prolong the battery's life. Of course, despite its overall advantage, lithium-ion battery has its drawbacks. It is fragile and requires a protection circuit to maintain safe operation [14]. Due to insufficient backup time, the super capacitor and flywheel are not applicable to the MV UPS.

\subsection{UPS transfer performance}

Tables 4 and 5 are load lists of each bus at normal operation and LOCA mode. The load capacity of the $01 \mathrm{~A}$ bus at the LOCA mode is largest.

Accordingly, test on the LOCA mode operation is required. Typical transfer performance of a MV UPS complying with the IEC $62040-3$ is as shown in Figure 6 $[13,15]$.

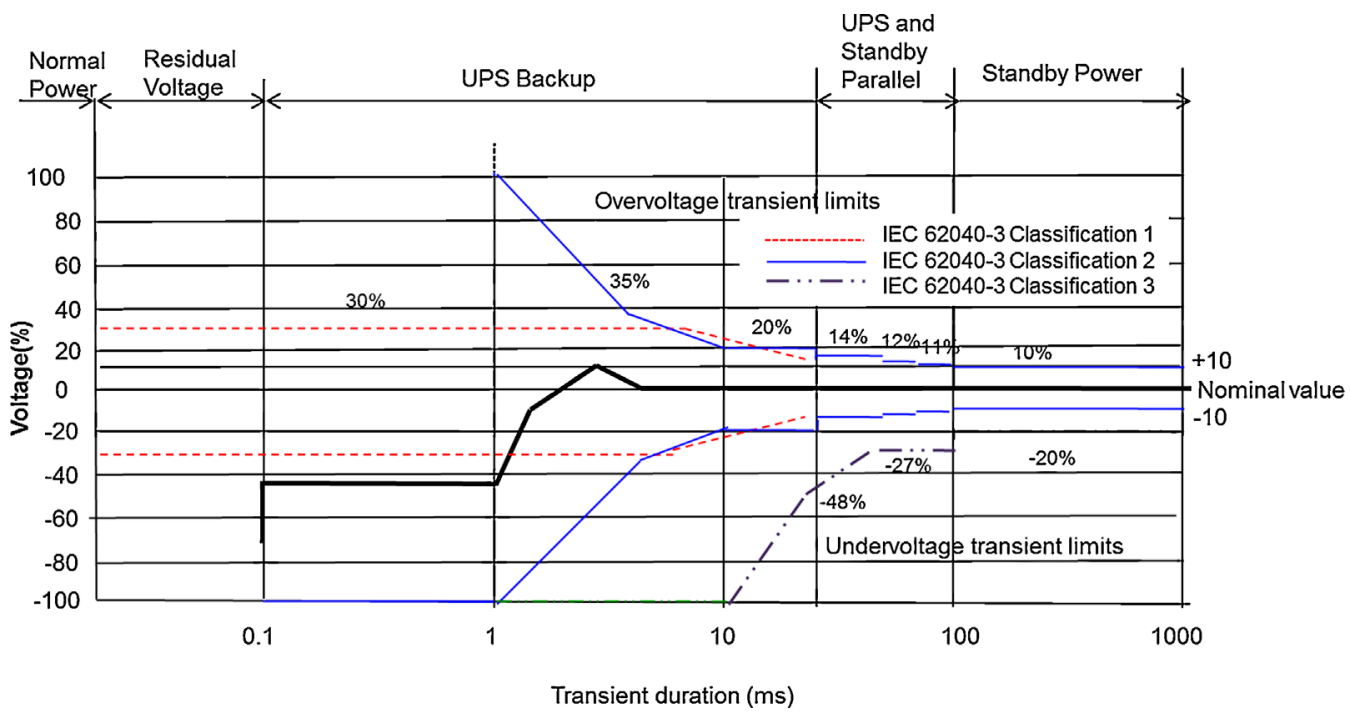

Fig. 6. Typical transfer performance of a MV UPS. 
Table 6. Test criteria.

\begin{tabular}{ll}
\hline Item & Specification \\
\hline $\begin{array}{l}\text { Transfer time (from normal } \\
\text { to inverter) }\end{array}$ & Less than $1.8 \mathrm{~ms}$ \\
$\begin{array}{l}\text { Output voltage setting time } \\
\text { (to within } \pm 10 \% \text { of set point) }\end{array}$ & Less than $5.0 \mathrm{~ms}$ \\
\hline
\end{tabular}

Table 7. Test facilities.

\begin{tabular}{ll}
\hline Item & Description \\
\hline Test date & April 2012 \\
Device under test & 180A 400 V 3PH + N Battery UPS-I \\
Software revision & R1E2 \\
Measurement & Multi Channel Hioki 8861 \\
method & Memory HiCORDER \\
Sag generator & IGBT instantaneous sag generator \\
\hline
\end{tabular}

Up until now, 10 MVA MV UPS has been developed and its test is ongoing. As an alternative, test results of the same type LV UPS are presented below. In the MV UPS system, the power converter and energy storage remain at LV with a transformer coupling these to MV. Therefore, it is expected that the MV UPS has the same performance as the same type LV UPS.

\subsubsection{Test configuration}

The following test results are typical performance of PCS-100 UPS-I product when a normal power failure event occurs. Results are shown for unity and inductive power factors. The two performance characteristics are presented in Tables 6 and 7 [16].

\subsubsection{Test results with inductive load (PF 0.5)}

Transfer characteristics under a 0.5 lagging power factor load are shown below. Even with 0.5 lagging power factor the UPS-I can transfer within $1.8 \mathrm{~ms}$ (actual transfer time is $1.48 \mathrm{~ms})$ (Fig. 7).

The Figure 8 is the same event as above, but processed in Excel to calculate the vector magnitude of the threephase overvoltage (purple line). The dashed lines indicate the $\pm 10 \%$ levels.

Vector magnitude $=\sqrt{2} \times$ maximum voltage.

The output voltage has settled to within $\pm 10 \%$ of nominal within $5.0 \mathrm{~ms}$ and is greater than $90 \%$ voltage after $3.0 \mathrm{~ms}$.

The MV UPS comply with the dynamic output performance classification of the IEC 62030-3 uninterruptible power systems (UPS) - Part 3: Method of specifying the performance and test requirements. Class 1 and Class 2 are applied to overvoltage and undervoltage respectively.

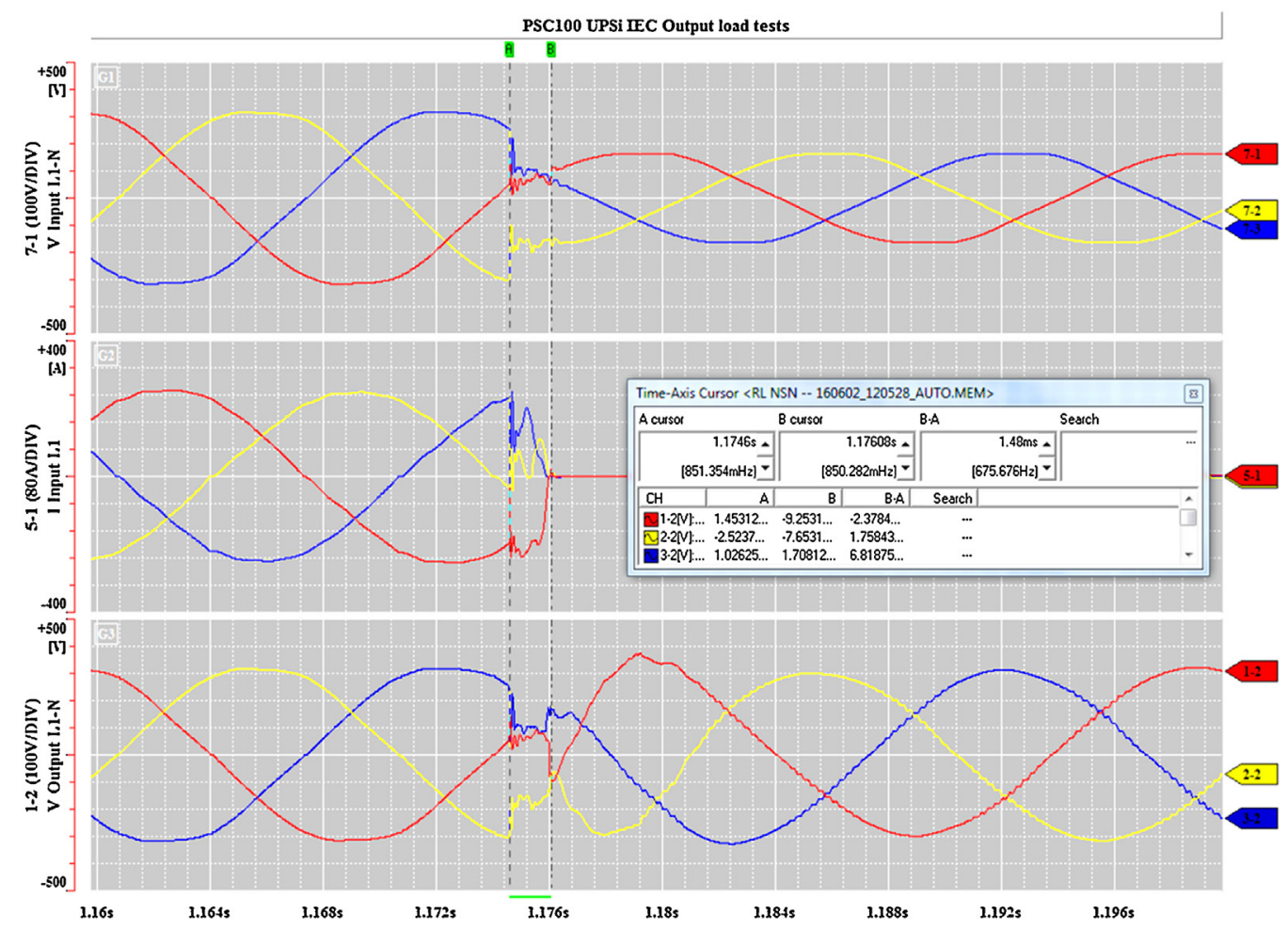

Fig. 7. UPS transfer characteristic curve. Top: input voltage; Mid: input current; Bottom: output voltage. 


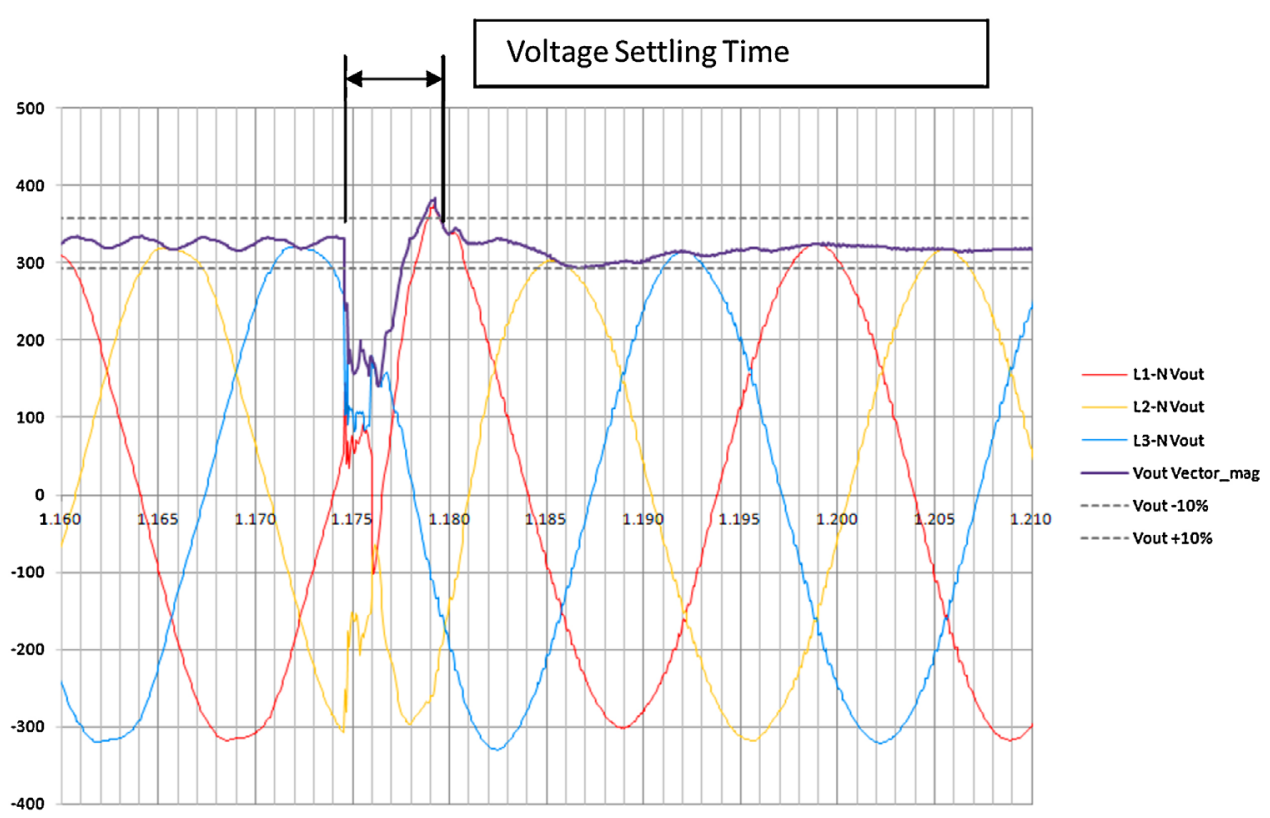

Fig. 8. Voltage settling time.

\section{Conclusion}

It takes $140 \mathrm{~ms}$ to transfer from normal to standby source after receiving starting request command in sequential fast transfer system. If the fast transfer has failed, residual transfer follows. Typical transfer time of residual transfer is 0.5 to 3 seconds. On the other hand, the transfer time of MV UPS is only $1.8 \mathrm{~ms}$ and output voltage setting time to within $\pm 10 \%$ of set point is $5.0 \mathrm{~ms}$.

Due to the various reasons specified in Table 1, fast bus transfer failure occurs occasionally in the existing system. However, the MV UPS can prevent such kind of failures except the failures caused by CB malfunction. In addition, the MV UPS makes the class 1E bus to transfer from normal source to the EDG seamlessly. It means that no load shedding and sequential loading are required to start up the EDG. During the severe accident condition when the LOOP and LOCA occur at the same time, the class $1 \mathrm{E}$ loads can operate continuously without interruption. Accordingly, the operator can shorten the countermeasure action time for accident with simplified operation procedure.

As a result, the uninterruptible MV bus transfer scheme improves the availability of the power of the safety bus and also safety of the nuclear power plant.

The size and price of the MV UPS varies with the autonomy time. According to the study, lead-acid battery is preferred for the purpose of short time backup (30 s; EDG starting time) and lithium-ion battery is preferred for the long time backup (10 min; AAC DG starting time). The latter one requires about $65 \%$ more space than the former one as shown in the Table 2 .

The effect of the safety enhancement, especially in the nuclear power plant, is scarcely converted to price. On the other hand, economic effect of the operation loss reduction in the specific power plant can be estimated based on the operation records. Further study will be conducted to evaluate the economic effect resulted from the proposed system before the deployment of the first uninterruptible MV bus transfer scheme to a nuclear power plant.

\section{Nomenclature}

$\begin{array}{ll}\text { AAC } & \text { alternative AC } \\ \text { CB } & \text { circuit breaker } \\ \text { EDG } & \text { emergency diesel generator } \\ \text { LV } & \text { low voltage } \\ \text { LOCA } & \text { loss of coolant accident } \\ \text { LOOP } & \text { loss of offsite power } \\ \text { MV } & \text { medium voltage } \\ \text { RCP } & \text { reactor coolant pump } \\ \text { SAT } & \text { standby auxiliary transformer } \\ \text { UAT } & \text { unit auxiliary transformer } \\ \text { UPS } & \text { uninterruptible power supply }\end{array}$

\section{References}

1. V. Nalamourougan, B. Kasztenny, A new high speed bus transfer relay design, implementation and testing, in Proceedings of the 2006 IEEE Power India Conference (2006)

2. IAEA Technical Report TR-271, Introducing nuclear power plant into electrical power systems of limited capacity: problems and remedial measures, 1987

3. International Atomic Energy Agency, Design of electric power systems for nuclear power plants, Draft Safety Guide DS-430, April 2012

4. R.D. Pettigrew, P. Powel, Motor bus transfer, IEEE Trans. Power Deliv. 8, 1747 (1993)

5. Siemens, 7VU683 High Speed Busbar Transfer Device, Chapter for the Catalog SIP, Edition No. 7, March 2014

6. M.V.V.S. Yalla, Design of a high-speed motor bus transfer system, IEEE Trans. Ind. Appl. 46, 612 (2010) 
7. A. Raje, A. Raje, J. McCall, A. Chaudhary, Bus transfer systems: requirements, implementation, and experiences, IEEE Trans. Ind. Appl. 39, 34 (2003)

8. M. Prasad, N. Theivarian, Normal power supply system of a nuclear power plant-modelling and simulation studies for fast bus transfer, in Proceedings of 2011 Electrical Energy System conference (IEEE, 2011), p. 294

9. M. Paliwal, R. Chandra Verma, S. Rastogi, Voltage sag compensation using dynamic voltage restorer, Adv. Electron. Electr. Eng. 4, 645 (2014)

10. L. Sevov, T.W. Zhao, I. Voloh, The power of IEC 61850, bustransfer and load-shedding applications, IEEE Ind. Appl. Mag. 19, 60 (2013)

11. Korea Institute of Nuclear Safety (KINS), Operation performance information system for nuclear power plant, accidents and events list, http://opis.kins.re.kr/index.jsp? Lan $=$ KR, Oct. 17, 2014

12. KHNP, NPP Systems, Auxiliary Power System, Text Book, No. BNP-FU-COM-SYS-TB-APS, Korea Hydro \& Nuclear Power Co. Rev. 2, p. 18

13. ABB, PCS100 Industrial Medium Voltage UPS Technical Proposal, No. 2UCD130469-T, Rev. A, 2013

14. Cadex Electronics Inc., Lithium-ion based batteries, http:// batteryuniversity.com/learn/article/lithium_based_bat teries, Aug. 19, 2015

15. Uninterruptible Power Systems (UPS). Part 3: Method of specifying the performance and test requirements, International Electrotechnical Commission, IEC 62040-3, 2011

16. ABB, ABB Technical Document, PCS100 UPS-I Transfer performance, Doc. No. 2UCD120000E023, 2014

Cite this article as: Choong-Koo Chang, A new MV bus transfer scheme for nuclear power plants, EPJ Nuclear Sci. Technol. 1, 12 $(2015)$ 